\title{
A Herschel study of Planetary Nebulae
}

\section{Griet C. Van de Steene ${ }^{1}$, Katrina M. Exter ${ }^{2}$, Peter A. M. van Hoof ${ }^{1}$, Tanya L. Lim ${ }^{3}$, Michael J. Barlow ${ }^{4}$, Mikako Matsuura ${ }^{4}$, Toshiya Ueta $^{5}$ and the MESS consortium}

\author{
${ }^{1}$ Royal Observatory of Belgium, Ringlaan 3, B-1180 Brussels, Belgium \\ ${ }^{2}$ IvS, Katholieke Universiteit Leuven, Celestijnenlaan 200 D, B-3001 Leuven, Belgium \\ ${ }^{3}$ Space Science and Technology Dept., Rutherford Appleton Lab., Oxfordshire OX11 0QX, UK \\ ${ }^{4}$ Dept. of Physics \& Astronomy, Univ. College London, Gower St, London WC1E 6BT, UK \\ ${ }^{5}$ Dept. of Physics and Astronomy, Univ. of Denver, Mail Stop 6900, Denver, CO 80208, USA
}

\begin{abstract}
We present Herschel PACS and SPIRE images of the dust shells around the planetary nebulae NGC 650, NGC 6853, and NGC 6720, as well as images showing the dust temperature in their shells. The latter show a rich structure, which indicates that internal extinction in the $\mathrm{UV}$ is important despite the highly evolved status of the nebulae.
\end{abstract}

Keywords. planetary nebulae: individual (NGC 650, NGC 6853, NGC 6720), infrared: ISM

\section{Introduction}

As part of the Herschel Guaranteed Time Key Project MESS (Mass loss of Evolved StarS) (PI Martin Groenewegen) we have imaged a sample of planetary nebulae (PNe) with the PACS (Poglitsch et al. 2010) and SPIRE (Griffin et al. 2010) instruments on board of the Herschel satellite (Pillbratt et al. 2010). A detailed description of the program can be found in Groenewegen et al. (2011) and an overview of the Herschel observations for PNe in van Hoof et al. (2012).

\section{Data Reduction}

All targets in this paper have been imaged in scan map mode. With PACS we have obtained images in the 70 and $160 \mu \mathrm{m}$ bands, with SPIRE in the 250, 350, and $500 \mu \mathrm{m}$ bands. PACS data were reduced up to level 1 within the data procession package HIPE (Ott 2010). The PACS images were made with the code Scanamorphos (Roussel 2011). The SPIRE images were reduced with the SPIRE pipeline. The images were convolved using the appropriate convolution kernels of Aniano et al. (2011) and rebinned to the pixel size of the longest wavelength image with flux conservation. These images were background subtracted before the ratios were taken. In order to convert the flux ratio images to temperature maps we determined the theoretical flux ratio at a given grain temperature by folding the grain emissivity of astronomical silicate or graphite (Martin \& Rouleau 1991) with the PACS and SPIRE filter transmission curves in HIPE using the procedure outlined in the SPIRE Observer's Manual. We interpolated the flux ratio as a function of temperature for each pixel in the the flux ratio image to obtain the temperature map. The temperature maps based on the ratio PACS 70 / PACS $160 \mu \mathrm{m}$ differs from PACS $160 /$ SPIRE $250 \mu \mathrm{m}$ by about $10 \mathrm{~K}$. The reason for this difference needs to be investigated further. Here we present the temperature maps based on the PACS 70 / PACS $160 \mu \mathrm{m}$ ratio images. 


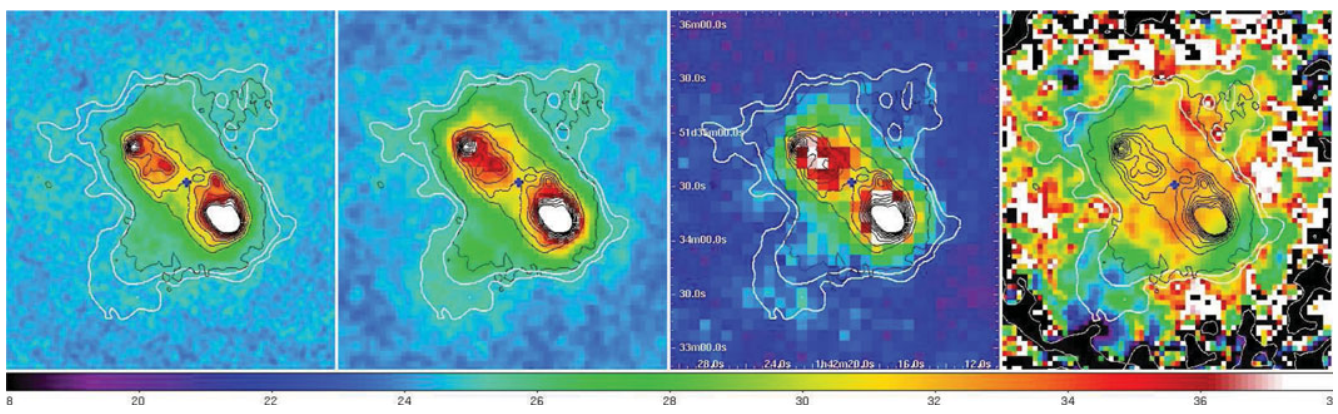

Figure 1. NGC 650, from left to right: PACS 70 and $160 \mu \mathrm{m}$, SPIRE $250 \mu \mathrm{m}$ and the temperature map created from the PACS $70 / 160 \mu \mathrm{m}$ ratio image. The black contours are of the PACS $70 \mu \mathrm{m}$ inner region and the white contours of the fainter outer regions of the PACS $160 \mu \mathrm{m}$ image. The blue cross marks the central star. The bar at the bottom shows the temperature scale.

\section{Results}

NGC 650 (the Little Dumbbell): in the temperature map of Fig. 1 the shadowing effect of the torus is clearly visible: the regions at the outer edge of the torus and beyond are clearly cooler than the dust in other directions. The dust grains are primarily heated by UV photons, either emitted by the central star, or diffuse emission from the gas (e.g., Ly $\alpha$ photons). Hence there must be substantial extinction of UV photons inside the torus. The measured flux values above $3 \sigma$ are $6.54,5.61$ and $1.27 \mathrm{Jy}( \pm 5 \%)$, at 70, 160, and $250 \mu \mathrm{m}$ respectively.

NGC 6853 (the Dumbbell): in the temperature map of Fig. 3 in van Hoof et al. (2012), we see a strong correlation between the high-density regions and the colder dust. The hot patch towards the south appears to be real and has no counterpart in the north. Presumably this is material that is directly irradiated by the central star. The measured flux values above $3 \sigma$ are 83.45 and $56.0 \mathrm{Jy}( \pm 5 \%)$ at 70 and $160 \mu \mathrm{m}$ respectively.

NGC 6720 (the Ring nebula): in Fig. 1 of van Hoof et al. (2012) we see that the halo is clearly detected in all 3 bands. The temperature map shows that the temperature is lower in the halo than inside the ring, because it is shielded from the starlight by the dense ring. The measured flux values above $3 \sigma$ are $54.70,29.18$ and $13.70 \mathrm{Jy}( \pm 5 \%$, at 70,160 , and $250 \mu \mathrm{m}$ respectively.

The similarity between the optical $\mathrm{H} \alpha$ and the FIR images, indicate that the gas and cool dust are well mixed in these objects.

The detailed match between the $\mathrm{H}_{2}$ emission and the FIR dust emission, suggests the formation of $\mathrm{H}_{2}$ on dust grains (van Hoof et al. 2010).

\section{References}

Aniano, G., Draine, B. T., Gordon, K. D., \& Sandstrom, K., 2011, PASP, 123, 1218

Griffin, M. J., Abergel, A., \& Abreu, A., et al., 2010, A\& A, 518, L3

Groenewegen, M. A. T., Waelkens, C., \& Barlow, M. J., et al., 2011, A\&\&A, 526, A162

Martin, P. G. \& Rouleau, F., 1991, in: F. Malina \& S. Bowyer eds., Extreme UV Astronomy, p. 341

Pilbratt, G. L., Riedinger, J. R., \& Passvogel, T., et al., 2010, A\&GA, 518, L1

Poglitsch, A., Waelkens, C., \& Geis, N., et al., 2010, A\&A, 518, L2

Ott, S., 2010, ASP Conference Series, 434, 139

Roussel, H., 2011, A\&A, submitted

van Hoof, P. A. M., Van de Steene, G. C., Barlow, M. J., et al., 2010, A\&گA, 518, 137

van Hoof, P. A. M., Barlow, M. J., Van de Steene, G. C., et al., 2012, these proceedings 